\section{P70 IMPROVED LUNG CANCER REFERRAL RATES AND EARLY DIAGNOSIS IN A DISTRICT GENERAL HOSPITAL}

MS Sidhu, AG Gulati, PH Hawkins, SC Cooper. Colchester General Hospital, Colchester, Essex, CO45JL, UK

\subsection{6/thoraxjnl-2014-206260.211}

Background Survival rates from lung cancer are poor in England with 5 year survival of $8 \%$ of men and $9 \%$ of women. ${ }^{1}$ We hypothesised that greater awareness of lung cancer symptoms resulting from local (2011) and national (2012) campaigns had increased our 2 week wait (2 WW) referrals and may have led to earlier diagnosis and better survival rates.

Methods We carried out a retrospective analysis of data between 2008 and 2013 of all patients referred with a new diagnosis of lung cancer. For all new referrals; the number of 2 week GP referrals, patient demographics, treatment modality, survival, and time to first outpatient appointment were examined.

Results 2 WW referrals increased by 26\% from 108 in 2008 to 421 in 2013, (peak 447 in 2012) and the number of confirmed lung cancer cases was 236 and 272 respectively, with 253 in 2012. 29.8\% of patients presented as an emergency in 2013 compared to $39.4 \%$ in 2008. During 2008 and 2010 4.2(mean) $[0.75](\mathrm{SD}) \%$ patients presented with stage IA disease compared to $6.3[0.7] \%$ during 2011 and 2013 ( $\mathrm{p}=0.05$ ). The rate of presentation with stage IV disease did not change significantly $(50 \%$ vs $56.6 \%, 2008$ and 2013 respectively). 15.1\% cases underwent curative treatment (surgical) in 2013 compared with 10.1\% in 2008. Survival at 6 and 12 months was unchanged. The time to first outpatient appointment was not significantly different (mean of 8 days).

Conclusion The peak of $2 \mathrm{WW}$ referrals coincided with local and national awareness campaigns. Although there was an increase in $2 \mathrm{WW}$ referrals this was not reflected in the number of confirmed lung cancer cases. However, there has been an

\section{Stage at diagnosis over a 6 year period presenting to the MDT}

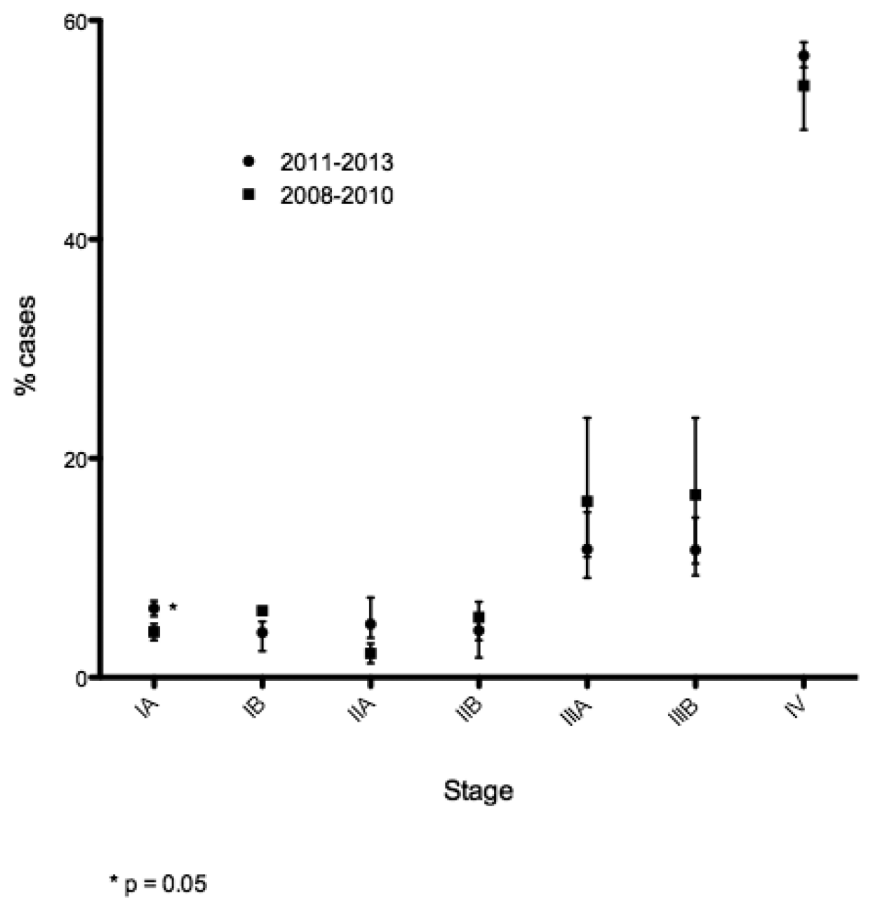

Abstract P70 Figure 1 Stage at diagnosis over a 6 period presenting to the MDT increase in the percentage of Stage IA diagnoses and resection rates which may have been a result of the campaigns increasing awareness amongst physicians and leading to earlier referrals.

\section{REFERENCE}

1 Office for National Statistics. Patients diagnosed 2005-2009 and followed up to 2010: Date accessed 12-07-2014. www.cancerresearchuk.org/cancer-info/cancerstats/types/lung/mortality/uk-lung-cancer-mortality-statistics

\section{\begin{tabular}{l|l}
\hline P71 POPULATION BASED EPIDEMIOLOGY, TREATMENT AND \\
\hline
\end{tabular} PROGNOSIS OF MALIGNANT MESOTHELIOMA IN LEEDS, UK - A MATCHED HISTORICAL COMPARISON}

RS Raju, MEJ Callister. St James's University Hospital, Leeds, UK

\subsection{6/thoraxjnl-2014-206260.212}

Introduction and objectives Since 2006 the National Lung Cancer Audit has provided increasingly comprehensive data on diagnosis, management and survival in patients with malignant mesothelioma on the UK. However, comparisons over a longer time period are hindered by a lack of comparable data. A previous population-based study of mesothelioma in Leeds, UK provided information on patients diagnosed between 2002 and 2005 (Thorax 2008;63:435). This current study describes the results of a matched analysis covering the years 2008-2013, allowing comparison with a historical cohort.

Methods 140 patients diagnosed with malignant pleural mesothelioma within the geographical boundaries of Leeds (CCG) were identified from 2008 to 2013 using Cancer Registry and National Lung Cancer Audit data. Clinical records from Leeds Teaching Hospitals $(n=136)$ or surrounding district hospitals $(n=4)$ were reviewed for information relating to disease characteristics at presentation, management and survival.

Results Clinical information for the current cohort is shown in Table 1 alongside data from the matched historical cohort from 2002-2005. The incidence per 100,000 population fell from 4.9

Abstract P71 Table 1 Comparison of data between previous study (2002-2005) and current study (2008-2013)

\begin{tabular}{lll}
\hline Parameter & $2002-2005$ & $2008-2013$ \\
\hline Number of cases & 146 & 140 \\
Incidence per 100,000 & 4.9 & 2.9 \\
Age - Median (Range) & $74(36-93)$ & $74(46-98)$ \\
Histological subtype & & \\
Epithelioid & $34(23.2 \%)$ & $99(70.7 \%)$ \\
Sarcomatoid & $10(6.8 \%)$ & $6(4.3 \%)$ \\
Biphasic & $9(6.1 \%)$ & $15(10.7 \%)$ \\
NOS & $93(63.7 \%)$ & $20(14.3 \%)$ \\
Pleural effusion management & & \\
Pleural effusion present & $110 / 146(75.3 \%)$ & $117 / 140(83.6 \%)$ \\
VATS/Thoracoscopic talc & $42 / 110(38.1 \%)$ & $51 / 117(43.6 \%)$ \\
IPC & $13 / 110(11.8 \%)$ & $26 / 117(22.2 \%)$ \\
Talc Slurry & $17 / 110(15.4 \%)$ & $1 / 117(0.9 \%)$ \\
& & \\
Oncological treatment & & $48 / 76(63.2 \%)$ \\
Port site radiotherapy & $73 / 122(59.8 \%)$ & $53 / 140(37.9 \%)$ \\
Chemotherapy (including trial) in all cases & $26 / 146(17.8 \%)$ & \\
Chemotherapy in patients with PS 0-2 & $26 / 92(28.2 \%)$ & $380(95 \% \mathrm{Cl} 252-397)$ \\
Median survival (days) & & \\
\hline
\end{tabular}


in 2002-2005 to 2.9 in 2008-2013. This partly reflects an increase in the population of the area covered over the time period (from 750,000 as described in the previous study to 806,683 in the current period).

Comparing earlier and later cohorts, there was a significant increase in the proportion of patients with a specific histological subtype $(36 \%$ vs $86 \%$ respectively, $\mathrm{p}<0.001)$ with a large rise in the proportion of epithelioid cases ( $32 \%$ vs $71 \%$ respectively). Similarly, comparing management of pleural effusion, the use of talc insufflation remained similar ( $38 \%$ vs $44 \%$ respectively) with increased use of indwelling pleural catheters (12\% vs $22 \%$ respectively) and a reduction in talc slurry pleurodesis $(15 \%$ vs $1 \%$ respectively). Overall treatment rates with palliative chemotherapy rose from $18 \%$ to $38 \%(\mathrm{p}=0.0002)$. Median survival rose from 267 days (95\% CI 178-356) to 380 days (95\% CI 252-397) between the two cohorts.

Conclusions The current study shows an unexpected reduction in measured incidence in Leeds, raising the possibility of incomplete case ascertainment in this study period. Specific histological subtyping, rates of palliative chemotherapy, and median survival increased between the cohorts.

\section{P72 INCIDENTAL DETECTION OF EARLY STAGE NON-SMALL CELL LUNG CANCER - TIME TO IMPLEMENT SCREENING?}

${ }^{1} \mathrm{RM}$ Thakrar, ${ }^{1} \mathrm{JM}$ Brown, ${ }^{2} \mathrm{SV}$ Brazil, ${ }^{3} \mathrm{M}$ Nankivell, ${ }^{4} \mathrm{DR}$ Lawrence, ${ }^{5} \mathrm{PJ}$ George, ${ }^{1} \mathrm{SM}$ Janes, ${ }^{5} \mathrm{~N}$ Navani. 'Lungs for Living Research Centre, University College London, UK; ${ }^{2}$ Department of Life Sciences, University College London, UK; ${ }^{3}$ MRC Clinical Trials Unit, London, UK; ${ }^{4}$ The Heart Hospital, London, UK; ${ }^{5}$ University College London Hospital, London, UK

\subsection{6/thoraxjnl-2014-206260.213}

Introduction Early detection is the key to survival in non-small cell lung cancer (NSCLC) where surgical resection can be undertaken. However, stage I and II disease combined account for only $25-30 \%$ of patients presenting with lung cancer. Although, clinical pathways from primary care exist to facilitate expeditious management of patients, the role of other referral pathways to diagnosis of surgically treatable lung cancers is not known.

Methods Patients suitable for surgical resection for curative intent for primary lung cancer were identified between 2007 and 2011 at this institution. Patients diagnosed 'incidentally' on radiology were compared to those detected through the standard

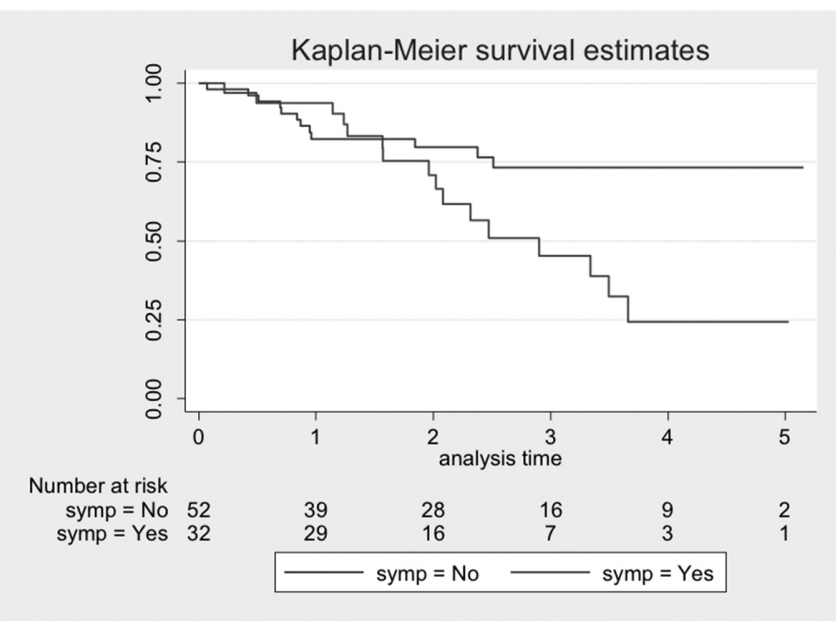

Abstract P72 Figure 1 Kaplan-Meier survival estimates 'two week' wait target referral system. Specific data on demographics, diagnostics utilised, pathological stage, and lung cancer mortality were recorded.

Results Eighty-four patients were treated with surgical resection for non-small cell cancer. The 'two-week' wait referrals accounted for one quarter ( $\mathrm{n}=21 ; 95 \%$ CI $17-35 \%)$ of the all the referrals, whilst $61 \%(\mathrm{n}=51 ; 95 \%$ CI $50-70 \%)$ of patients were found to have lung cancer detected incidentally through investigations performed by other specialties in the hospital. The presentation of patients to hospital with cancer related symptoms whether they had operable disease or not, had significantly higher lung cancer specific mortality ( $p=0.02$; see figure).

Conclusion We demonstrate that patients who have cancerrelated symptoms have a worse outlook. Whilst asymptomatic patients diagnosed by chance have better prospects for cure by surgical resection, thus highlighting the promise of CT screening for lung cancer in patients with high risk factors.

\section{P73 THE RATE OF INCIDENTAL SYNCHRONOUS PATHOLOGY ON PET-CT SCANS PERFORMED FOR THORACIC MALIGNANCY AND SUBSEQUENT IMPACT ON LUNG CANCER PATHWAYS}

TRE Jones, HJ Curtis. Queen Elizabeth Hospital, Gateshead, UK

\subsection{6/thoraxjnl-2014-206260.214}

Introduction NICE (CG121) recommends that all patients potentially suitable for curative intent treatments are offered PET-CT, and that they are treated within 62 days of their urgent referral and within 31 days of the decision to treat.

There are case reports and three large studies regarding incidental findings on PET-CT performed for thoracic malignancy. These studies were based in Sheffield, Australia and Switzerland, with a rate of significant incidental findings of $21 \%, 12 \%$ and $9 \%$ respectively. There are no studies regarding the impact on referral pathways.

We aimed to identify the rate of incidental synchronous pathology on PET-CT for thoracic malignancy in our local population of 190,000 and the impact of these on referral pathways. We serve a local authority district ranked 43/326 in the English Index Multiple Deprivation in 2010 (rank of 1 being most deprived).

Methods Identifying patients from our thoracic MDT database, we retrospectively analysed electronic patient records for those with synchronous pathology on PET-CT between November 2012 and October 2013. Data collected included primary

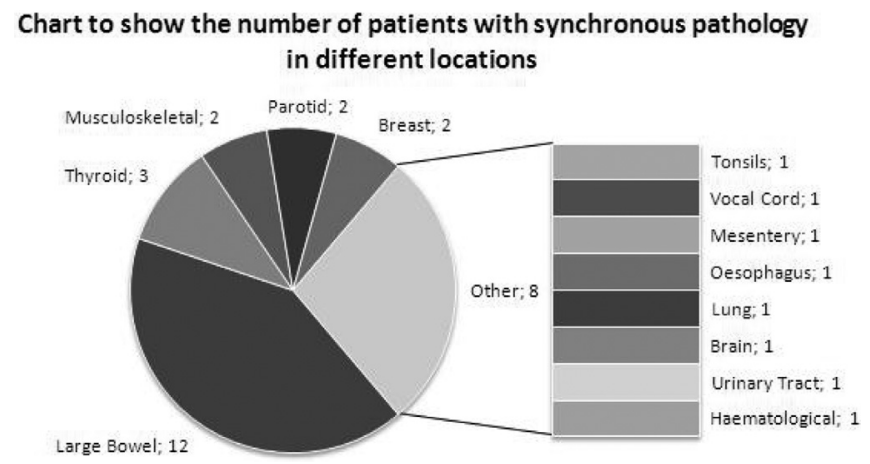

Abstract P73 Figure 1 Chart to show the number of patients with synchronous pathology in different locations 